University of New Orleans

ScholarWorks@UNO

10-1-1995

\title{
Extreme ultraviolet polarizing optics using bare and aluminum- coated silicon carbide
}

\author{
R. M.A. Azzam \\ University of New Orleans, razzam@uno.edu
}

A. M. Kan'an

Follow this and additional works at: https://scholarworks.uno.edu/ee_facpubs

Part of the Electrical and Electronics Commons

\section{Recommended Citation}

R. M. A. Azzam and A. M. Kan'an, "Extreme ultraviolet polarizing optics using bare and aluminum-coated silicon carbide," Appl. Opt. 34, 6438-6442 (1995)

This Article is brought to you for free and open access by the Department of Electrical Engineering at ScholarWorks@UNO. It has been accepted for inclusion in Electrical Engineering Faculty Publications by an authorized administrator of ScholarWorks@UNO. For more information, please contact scholarworks@uno.edu. 


\title{
Extreme ultraviolet polarizing optics using bare and aluminum-coated silicon carbide
}

\author{
R. M. A. Azzam and A. M. Kan'an
}

\begin{abstract}
A deformable three-reflection system that uses a bare silicon carbide substrate can function as an in-line, high-throughput $(>30 \%), 90^{\circ}$ phase shifter in the $50-100 \mathrm{~nm}$ spectral range. For a given extreme ultraviolet wavelength, an aluminum thin film can be deposited on the silicon carbide substrate to suppress the parallel $(p)$ or perpendicular $(s)$ polarization on single reflection or to introduce quarter-wave retardation and equal reflectances for incident $p$ - and $s$-polarized light.
\end{abstract}

\section{Introduction}

Because of the lack of suitable transparent birefringent materials, reflection-type polarization optical devices are important in the vacuum ultravioletextreme ultraviolet (VUV-EUV) and soft-x-ray spectral regions. One advantage of reflection devices is that mirror materials that are stable in the presence of EUV radiation can be used. (If a transparent or semitransparent birefringent material exists, such as $\mathrm{MgF}_{2}$ in the 120-200-nm range, its performance may be affected by radiation damage to the medium.

The use of in-line (insertion) reflection optical devices to convert in-plane-of-orbit synchrotron radiation from linear to circular polarization has received considerable attention recently (see, e.g., Refs. 1-4), and researchers are looking for mirror materials that can be used to achieve relatively high throughput. The same optical devices are also essential for the analysis or measurement of arbitrary elliptical polarization states (i.e., as components of Stokes parameter photopolarimeters). 5

Silicon carbide $(\mathrm{SiC})$ is a promising new material for reflection optics in the EUV spectral region because of high normal-incidence reflectance (30-50\% in the 60-200-nm wavelength range), durability, and low coefficient of thermal expansion.,7 From the optical constants of $\mathrm{SiC}$ reported by Windt et al., ${ }^{8}$ we

When this research was performed the authors were with the Department of Electrical Engineering, University of New Orleans, New Orleans, Louisiana 70148. A. M. Kan'an is now with the Center for Research in Electro-Optics and Lasers, University of Central Florida, Orlando, Florida 32826.

Received 5 December 1994; revised manuscript received 29 March 1995.

0003-6935/95/286438-05\$06.00/0.

(1) 1995 Optical Society of America. show that $\mathrm{SiC}$ mirrors can be used in a deformable three-reflection system as a $90^{\circ}$ phase shifter with a relatively high throughput $(>30 \%)$ in the $50-100-\mathrm{nm}$ spectral region. This throughput is higher than that attainable with any metal. 1,9 We also show that, at a given wavelength, the $p$ or $s$ polarization can be suppressed on single reflection by the use of light interference in an $\mathrm{Al}$ thin film deposited on the $\mathrm{SiC}$ substrate. Furthermore, a quarter-wave retarder (QWR) without diattenuation (i.e., with equal throughput for the $p$ and $s$ polarizations) can be designed by the use of the same Al-SiC film-substrate system.

\section{In-Line Three-Reflection Quarter-Wave Retarder}

An in-line three-reflection QWR is a device that introduces a relative phase shift of $90^{\circ}$ between the $p$ and $s$ components of the incident monochromatic light. Incident linearly polarized light of the appropriate azimuth is converted to circularly polarized light when the $p$ and $s$ components of the emergent light have equal amplitudes.

The ellipsometric function, $\rho$, is defined as the ratio $r_{p} / r_{s}$ of the $p$ and $s$ complex amplitude-reflection coefficients and is usually written in the form $\rho=$ $\tan \psi \exp (j \Delta)$, where $\tan \psi$ is the $p$-to-s relative amplitude attenuation and $\Delta$ is the $p$-to-s relative phase shift on reflection. ${ }^{10}$ We consider monochromatic linearly polarized light that is incident at an angle $\phi$ with its electric field vibration at an azimuth $\theta$ from the plane of incidence. For a given mirror material with complex dielectric constant $\varepsilon$, a cumulative differential phase shift of $90^{\circ}$ (or an odd multiple thereof) can be achieved by an appropriate choice of the angle of incidence $\phi$, called the principal angle and denoted by $\bar{\phi}$. The value of $\theta$ required to produce output circularly polarized light at $\bar{\phi}$ is called the principal azimuth and is denoted by $\bar{\psi}$. 


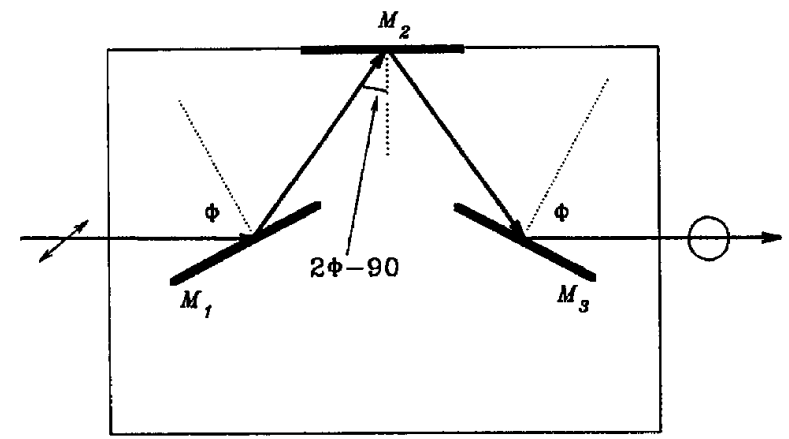

Fig. 1. Geometry of the in-line three-reflection phase retarder.

Figure 1 shows the geometry of the in-line threereflection system. The three mirrors are assumed to be polished surfaces of the same substrate material, and the angles of incidence at the first and the third mirrors are equal because of symmetry. The angle of incidence at the second mirror is equal to $2 \phi-90^{\circ}$. The entire system is rotatable around the incident light beam as an axis.

The net ellipsometric function is given by

$$
\rho_{n}=\rho^{2}(\phi) \rho\left(2 \phi-90^{\circ}\right)
$$

where

$$
\rho(\phi)=\frac{\sin \phi \tan \phi-\left(\varepsilon-\sin ^{2} \phi\right)^{1 / 2}}{\sin \phi \tan \phi+\left(\varepsilon-\sin ^{2} \phi\right)^{1 / 2}} .
$$

For this three-reflection system to introduce a $\pm 90^{\circ}$ differential phase shift, the real part of $\rho_{n}$ is set equal to zero so that $\rho_{n}=\tan \bar{\psi} \exp ( \pm j \pi / 2)$. The net intensity transmittance, or the overall throughput of the system, when operated as a linear-to-circular polarization transformer, is given by

$$
T=\mathscr{T}_{p n} \cos ^{2} \bar{\psi}+\mathscr{T}_{s n} \sin ^{2} \bar{\psi}
$$

where

$$
\begin{aligned}
& \mathscr{M}_{v n}=\left|r_{v n}\right|^{2}, \\
& r_{v n}=r_{v}{ }^{2}(\bar{\phi}) r_{v}\left(2 \bar{\phi}-90^{\circ}\right), \quad v=p, s .
\end{aligned}
$$

To determine $\bar{\phi}$ analytically is difficult and cumbersome; therefore, the principal angle and principal azimuth are calculated numerically. Note from Fig. 1 that $\bar{\phi}$ cannot be $<45^{\circ}$.

The optical data reported by Windt et al. ${ }^{8}$ are used to calculate the principal angle and the principal azimuth when chemical vapor deposition $(\mathrm{CVD}) \mathrm{SiC}$ is used as the mirror material. Figure 2 shows $\bar{\phi}$ as a function of $\lambda$ in the VUV and EUV region. There is more than one solution for the principal angle at each wavelength $\lambda>10 \mathrm{~nm}$. The cumulative differential phase shifts for the three solutions of $\bar{\phi}_{0}, \bar{\phi}_{1}$, and $\bar{\phi}_{2}$ are $270^{\circ}, 270^{\circ}$, and $90^{\circ}$, respectively. The largest principal angle, $\bar{\phi}_{2}$, is the more important, because the overall throughput at this angle is larger than it is at the other principal angles. The net intensity transmittance at $\bar{\phi}_{0}$ and $\bar{\phi}_{1}$ is small $(<10 \%)$.

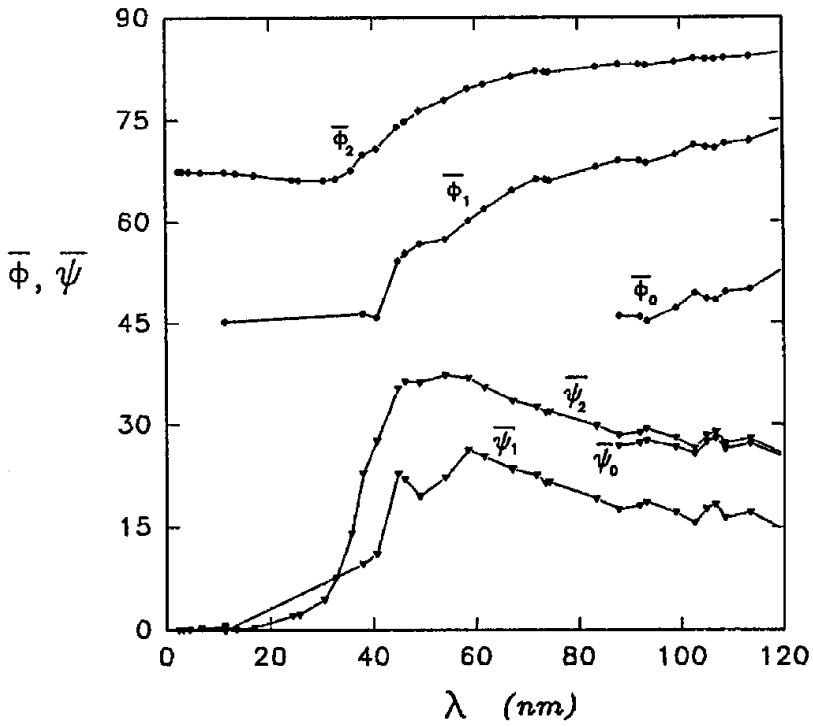

Fig. 2. Principal angles $\bar{\phi}_{i}$ and principal azimuths $\bar{\psi}_{i}(i=0,1,2)$ for CVD SiC mirrors as functions of $\lambda$ in the VUV-EUV spectral region. The optical constants of $\mathrm{SiC}$ are taken from Ref. 8.

Figure 3 shows intensity transmittance $T$ of the system calculated at $\bar{\phi}_{2}$. In general, the higher the principal azimuth, the higher the throughput, which is consistent with Eq. (3) because $\left|r_{s}\right|>\left|r_{p}\right|$. To compare the calculated values of $T$ for CVD $\mathrm{SiC}$ with those for Au (which is commonly used), we take the optical constants of Au also from Ref. 8. Within the same spectral region, $T$ for $\mathrm{Au}$ is $<T$ for $\mathrm{SiC}$ by a factor of 3 or more. For example, at the He I wavelength of $58.43 \mathrm{~nm}, T$ of the $\mathrm{SiC}$ system is $44.7 \%$ whereas that of Au is only $8 \%$.

\section{Reflection Polarizers and Phase Retarders that Use an Absorbing Thin Film on an Absorbing Substrate}

Single-reflection retarders that use a transparent thin film on an absorbing substrate have been de-

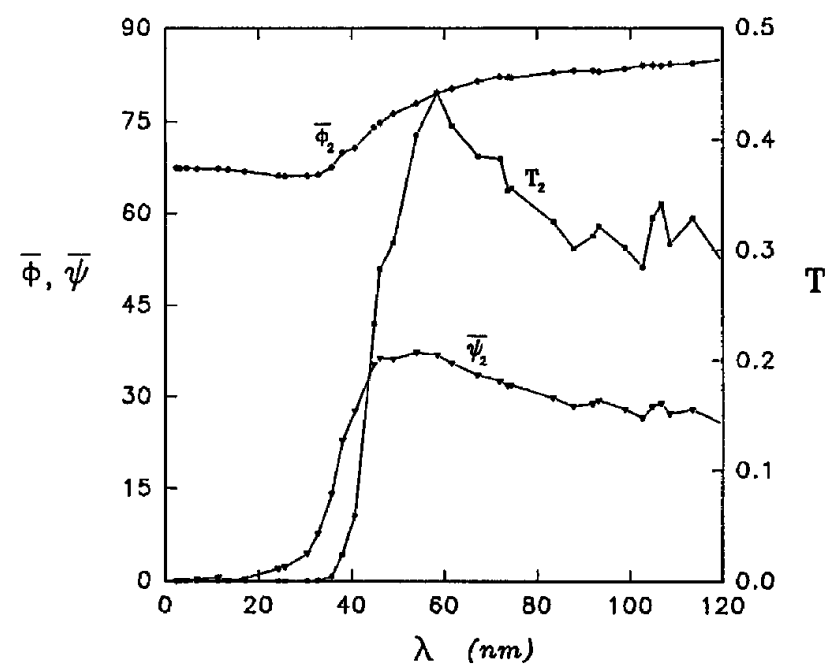

Fig. 3. Largest principal angle $\bar{\phi}_{2}$, associated principal azimuth $\bar{\psi}_{2}$, and throughput $T_{2}$ for a CVD SiC three-mirror system as functions of $\lambda$.

1 October 1995 / Vol. 34, No. 28 / APPLIED OPTICS 
scribed by Azzam and co-workers. ${ }^{11,12}$ Thonn and Azzam ${ }^{13}$ described dielectric-coated metallic mirrors for fixed three-reflection phase retarders in the IR spectral region, and Azzam and Kan'an ${ }^{14}$ obtained variable-phase retardation in the IR by using a deformable three-mirror system with $\mathrm{ZnS}$-coated Ag mirrors.

The change of polarization on single reflection from an optically isotropic surface is completely determined by $\rho$. For a light beam of wavelength $\lambda$ incident at an angle $\phi$ from a transparent ambient (with $\left.\varepsilon=\varepsilon_{0}\right)$ onto an absorbing substrate $\left(\varepsilon=\varepsilon_{2}\right)$, which is coated with an absorbing film of thickness $d$ and $\varepsilon=\varepsilon_{1}$, the $p$ and $s$ reflection coefficients are given by $^{10}$

$$
r_{v}=\frac{r_{01 v}+r_{12 v} X_{1}}{1+r_{01 v} r_{12 v} X_{1}}, \quad v=p, s
$$

In Eq. (5), $r_{01 v}$ and $r_{12 v}$ are the ambient-film and film-substrate Fresnel interface reflection coefficients for the $v$ polarization:

$$
\begin{aligned}
r_{i j p} & =\frac{\varepsilon_{j} S_{i}-\varepsilon_{i} S_{j}}{\varepsilon_{j} S_{i}+\varepsilon_{i} S_{j}}, \quad r_{i j s}=\frac{S_{i}-S_{j}}{S_{i}+S_{j}}, \quad i j=01,12, \\
X_{1} & =\exp \left(\frac{-j 4 \pi d}{\lambda} S_{1}\right), \\
S_{i} & =\left(\varepsilon_{i}-\varepsilon_{0} \sin ^{2} \phi\right)^{1 / 2}, \quad i=0,1,2 .
\end{aligned}
$$

Because $\varepsilon_{1}$ of the film is complex, $S_{1}$ is also complex in general. For a given film thickness $d$, as $\phi$ is increased from zero (normal incidence) to $90^{\circ}$ (grazing incidence), $\rho$ traces a trajectory in the complex plane that starts at $(-1+j 0)$ for $\phi=0$ and ends at $(1+j 0)$ for $\phi=90^{\circ}$. For $d=0$ the contour is that of the bare substrate, whereas for large $d$ (thick film), $\rho$ traces a curve that is characteristic of bulk film material because light does not penetrate to the film-substrate interface). To increase the range of $d$ in which the film is semitransparent, one must make sure that the film material has a small extinction coefficient.

Consider a $\mathrm{SiC}$ substrate $\left[\varepsilon_{2}=(0.401-j 0.52)^{2}\right]$ that is coated with a thin film of $\mathrm{Al}\left[\varepsilon_{1}=(0.796-j 0.0959)^{2}\right]$ at the He I wavelength $\lambda=58.43 \mathrm{~nm}$. Here $\rho$ is calculated at different thicknesses $d$ as $\phi$ increases from 0 to $90^{\circ}$. In Fig. 4 these constant thickness contours (CTC's) are shown for $d=0,32-42 \mathrm{~nm}, \infty$. The spiralling constant angle of incidence (variablethickness) contours (CAIC's) are also shown in the same figure for $\phi=15-75^{\circ}$ in steps of $15^{\circ}$. Each CAIC starts on the $d=0 \mathrm{CTC}$ and ends spirally on the $d=\infty$ contour. The spiralling is due to the low film absorption. The $\phi=30^{\circ}$ CAIC spans a large region of the complex plane. As $d$ increases from zero, the CTC expands out in the upper half-plane. This is seen for $d=32 \mathrm{~nm}$ and $d=34 \mathrm{~nm}$. Increasing $d$ further causes $\rho$ to go to $\infty$ and then come

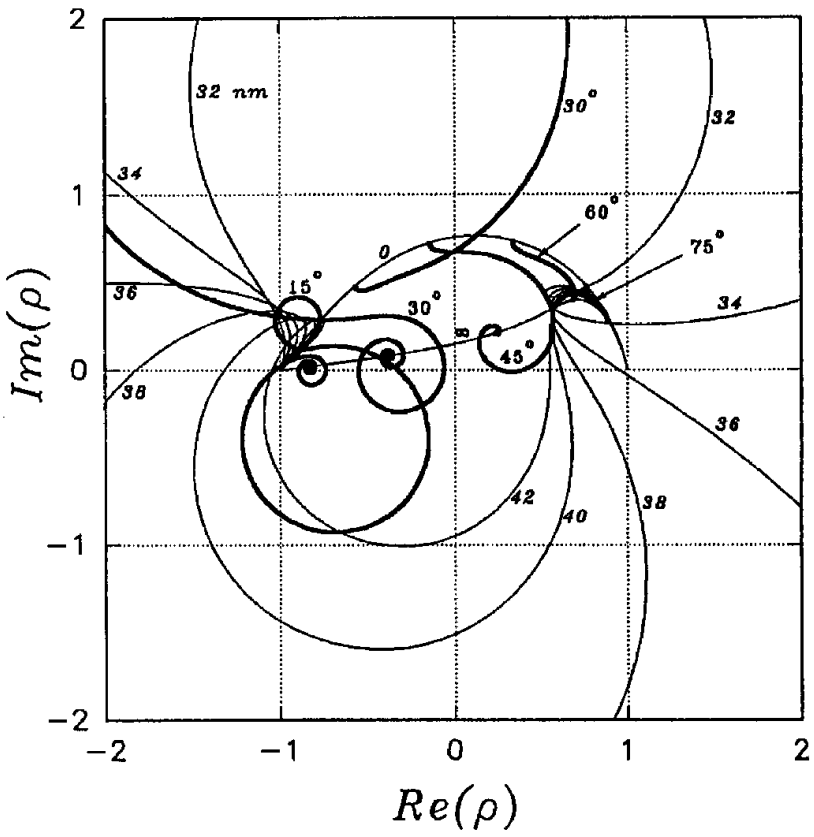

Fig. 4. Contours of constant film thickness $d$ and constant angle of incidence $\phi$ in the complex $\rho$ plane for single reflection by an $\mathrm{Al}-\mathrm{SiC}$ film-substrate system at $\lambda=58.43 \mathrm{~nm}$.

back in the lower half-plane. Most of the $\rho$ complex plane is covered. Therefore, essentially any desired value can be assigned to $\rho$ by means of the control of $d$ and $\phi$. To make a QWR with $\rho=j$, we look for $d$ that is $<32 \mathrm{~nm}$. Making a QWR with $\rho=-$ requires a thickness of $38 \mathrm{~nm}<d<42 \mathrm{~nm}$. To make a $p$-suppressing reflection polarizer with $\rho=0$, one can
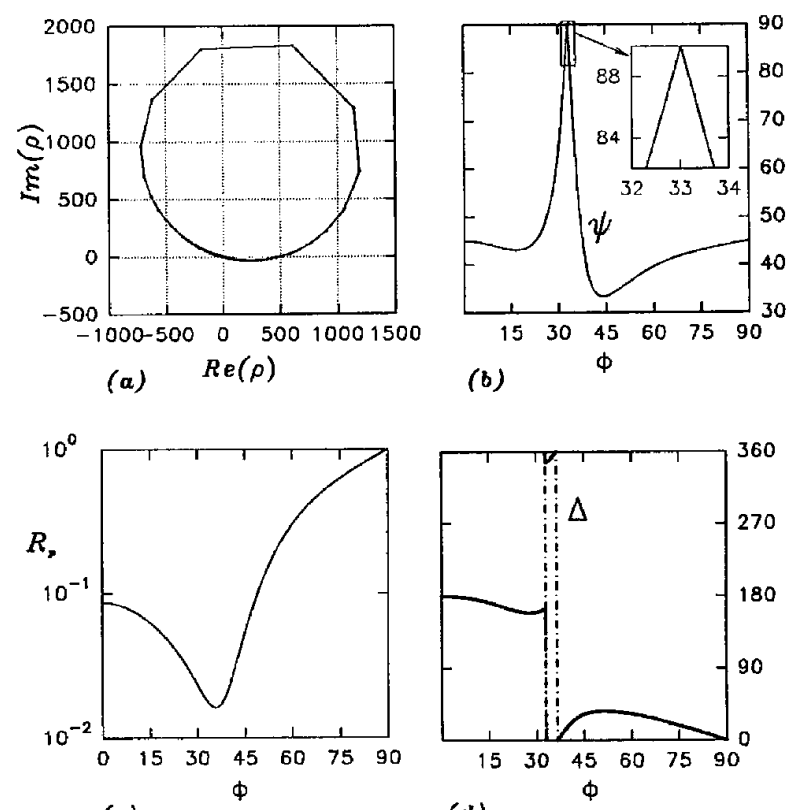

(a)

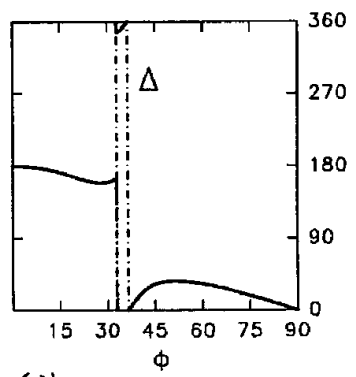

(d)

Fig. 5. (a) $\operatorname{Re}(\rho)$ versus $\operatorname{Im}(\rho)$ as angle of incidence $\phi$ is changed from $0^{\circ}$ to $90^{\circ}$ for the Al-SiC film-substrate $s$-suppressing singlereflection polarizer at $\lambda=58.43 \mathrm{~nm}$. (b) Ellipsometric angle $\psi$ versus $\phi$. (c) Intensity reflectance $R_{p}$ versus $\phi$. (d) Differential reflection phase shift $\Delta$ versus $\phi$. 

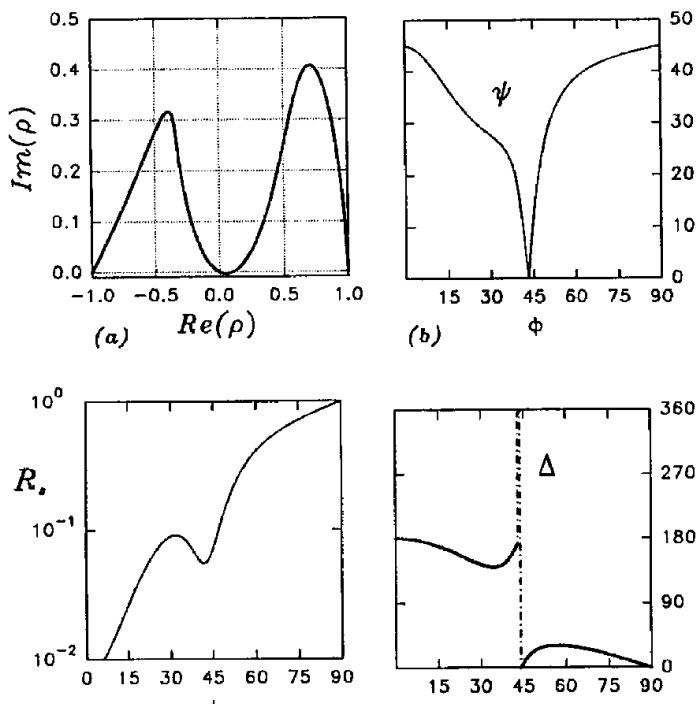

(c)

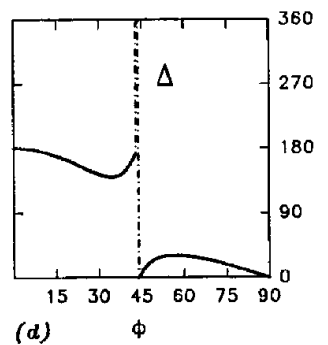

Fig. 6. (a) $\operatorname{Re}(\rho)$ versus $\operatorname{Im}(\rho)$ as angle of incidence $\phi$ is changed from $0^{\circ}$ to $90^{\circ}$ for the $\mathrm{Al}-\mathrm{SiC}$ film-substrate $p$-suppressing singlereflection polarizer at $\lambda=58.43 \mathrm{~nm}$. (b) Ellipsometric angle $\psi$ versus $\phi . \quad$ (c) Intensity reflectance $R_{p}$ versus $\phi$. (d) Differential reflection phase shift $\Delta$ versus $\phi$.

find a value of $d>42 \mathrm{~nm}$. Finally, to cause $\rho$ to go to $\infty$ (an $s$-suppressing reflection polarizer), we search for a value of $d$ between 34 and $36 \mathrm{~nm}$.

To suppress the $s$ component in reflection (i.e., $r_{s}=0$ and $\left.\rho \rightarrow \infty\right)$ we find $d=35.12 \mathrm{~nm}$ and $\phi=$ $33.03^{\circ}$. For this thickness, the contour of $\rho$ is shown in Fig. $5(\mathrm{a})$ and is nearly a circle. Figure $5(\mathrm{~b})$ shows $\psi$ as a function of $\phi$; it is $90^{\circ}$ at $\phi=33.03^{\circ}$. The intensity reflectance, $R_{p}$, is shown in Fig. 5(c) as a function of $\phi$; it is 0.018 at $\phi=33.03^{\circ}$. Finally, $\Delta$ is
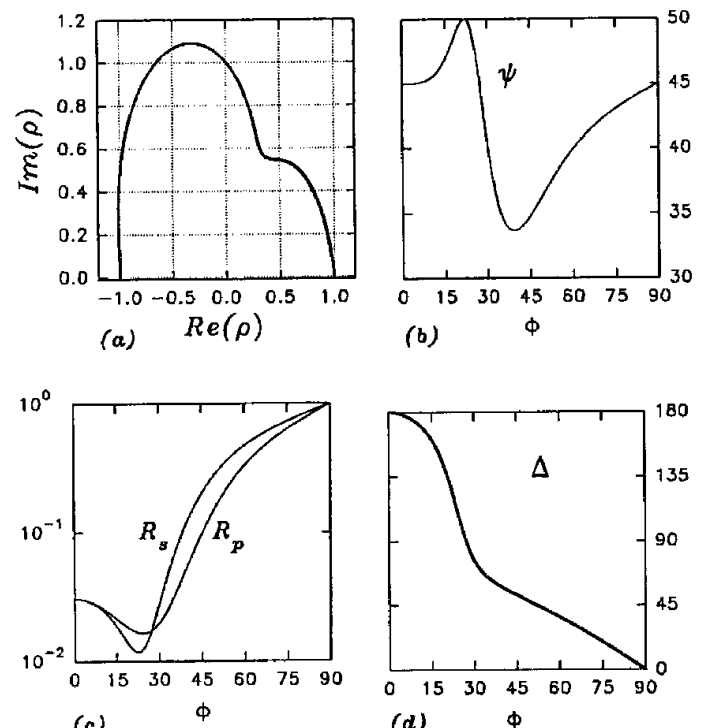

(c)

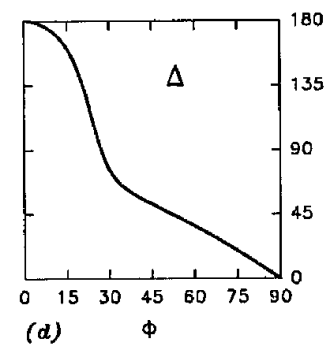

Fig. 7. (a) $\operatorname{Re}(\rho)$ versus $\operatorname{Im}(\rho)$ as angle of incidence $\phi$ is changed from $0^{\circ}$ to $90^{\circ}$ for the Al-SiC film-substrate single-reflection QWR $(\rho=j)$ at $\lambda=58.43 \mathrm{~nm}$. (b) Ellipsometric angle $\psi$ versus $\phi$. (c) Intensity reflectances $R_{p}$ and $R_{s}$ versus $\phi$. (d) Differential reflection phase shift $\Delta$ versus $\phi$. shown in Fig. 5(d). Note the fast change in $\Delta$ around the polarizing angle of $33.03^{\circ}$.

To make $r_{p}=0$, we determine $d=55.8 \mathrm{~nm}$ and $\phi=$ $43.9^{\circ}$. The $\rho$ contour, $\psi, R_{p}$, and $\Delta$ are all shown in Figs. 6(a), 6(b), 6(c), and 6(d), respectively. At the polarizing angle of $43.9^{\circ}, R_{s}=0.057$. This reflectance level is adequate for certain applications.

Finally, we consider the design of a pure QWR so that $\rho= \pm j$. The solutions for the film thicknesses are 41.74 and $27.07 \mathrm{~nm}$ for $\rho=-j$ and $j$, respectively. We take the lesser thickness because the associated reflectance is larger. The angle at which $\rho=j$ for $d=27.07 \mathrm{~nm}$ is $27.2^{\circ}$. As the angle of incidence $\phi$ is changed, the $\rho$ contour, $\psi, R_{v}$, and $\Delta$ are shown in Fig. 7. At $\phi=27.2^{\circ}, R_{p}=R_{s}=1.7 \%$. An error of $\pm 2 \mathrm{~nm}$ in the film thickness causes a corresponding error in the differential phase shift of $<8^{\circ}$.

\section{Conclusions}

We have shown that by using CVD $\mathrm{SiC}$ mirrors, one realizes a three-reflection $90^{\circ}$ phase retarder with largely increased throughput. This improvement is quite important in the EUV spectral region, where no transparent birefringent materials exist. In addition, this device is inexpensive when compared with more complicated designs that employ multilayer coatings. A pure QWR (without diattenuation) can be achieved on single reflection by an $\mathrm{Al}$-coated $\mathrm{SiC}$ mirror at the expense of a reduction in reflectance.

Theoretically ideal interference-based single-reflection polarizers with low throughput for the unextinguished polarization (of the order of a few percent) are designed by the use of the $\mathrm{Al}-\mathrm{SiC}$ film-substrate system at the He I EUV wavelength of $58.43 \mathrm{~nm}$.

It should be noted that we have assumed perfectly smooth and clean optics throughout this paper, i.e., the absence of possible contamination (e.g., carbon) or oxide films on the plane surfaces of the bare or coated $\mathrm{SiC}$ mirrors. The actual performance of devices that are constructed according to the designs described here may be significantly affected by such films and by surface roughness.

This research was presented at the Annual Meeting of the Optical Society of America, 2-7 October 1994, in Dallas, Texas.

\section{References}

1. P. D. Johnson and N. V. Smith, "Production of circularly polarized light from synchrotron radiation in the vacuum ultraviolet region," Nucl. Instrum. Methods 214, 505-508 (1983).

2. T. Döhring, G. Schönhense, and U. Heinzmann, "A circular polarizer for the region of windowless VUV radiation," Meas. Sci. Technol. 3, 91-97 (1992).

3. T. Koide, T. Shidara, M. Yuri, N. Kandaka, and H. Fukutani, "Production and direct measurement of circularly polarized vacuum-ultraviolet light with multireflection optics," Appl. Phys. Lett. 58, 2592-2594 (1991).

4. T. Saito, A. Ejiri, and H. Onuki, "Polarization properties of an evaporated aluminum mirror in the VUV region," Appl. Opt. 29, 4538-4540 (1990).

5. T. Koide, T. Shidara, M. Yuri, N. Kandaka, H. Fukutani, and 
K. Yamagutchi, "Elliptical polarization measurements in the vacuum ultraviolet and soft x-ray regions with a reflection polarimeter," Rev. Sci. Instrum. 63, 1458-1461 (1992).

6. G. E. Holland, J. F. Seely, R. P. McCoy, K. F. Dymond, C. Rollins, and M. Corson, "Effect of energetic electron and proton bombardment on the reflectance of silicon carbide mirrors in the extreme-ultraviolet region," Appl. Opt. 33, 5902-5905 (1994).

7. J. B. Kortright and D. C. Windt, "Amorphous silicon carbide for extreme ultraviolet optics," Appl. Opt. 27, 2841-2846 (1988).

8. D. L. Windt, W. C. Cash, Jr., M. Scott, P. Arendt, B. Newnam, R. F. Fisher, and A. B. Swartzlander, "Optical constants for thin films of Ti, Zr, Nb, Mo, Ru, Rh, Pd, Ag, Hf, Ta, W, Re, Ir, Os, Pt, and Au from $24 \AA$ to $1216 \AA$," Appl. Opt. 27, 246-278 (1988); D. L. Windt, W. C. Cash, Jr., M. Scott, P. Arendt, B. Newnam, R. F. Fisher, A. B. Swartzlander, P. Z. Takacs, and J. M. Pinneo, "Optical constants for thin films of C, diamond, $\mathrm{Al}, \mathrm{Si}$, and CVD SiC from $24 \AA$ to $1216 \AA$ Å, Appl. Opt. 27, 279-295 (1988).

9. A. M. Kan'an and R. M. A. Azzam, "Principal angles and principal azimuths of an in-line symmetric three-reflection bare-mirror system: application to circular polarization of VUV radiation," in Polarization Analysis and Measurement II, D. H. Goldstein and D. B. Chenault, eds., Proc. Soc. Photo-Opt. Instrum. Eng. 2265, 9-14 (1994).

10. R. M. A. Azzam and N. M. Bashara, Ellipsometry and Polarized Light (North-Holland, Amsterdam, 1987), Chap. 4.

11. R. M. A. Azzam, A. R. M. Zaghloul, and N. M. Bashara, "Ellipsometric function of a film-substrate system: applications to the design reflection-type optical devices and to ellipsometry," J. Opt. Soc. Am. 65, 252-260 (1975).

12. R. M. A. Azzam and B. E. Perilloux, "Constraint on the optical constants of a film-substrate system for operation as an external-reflection retarder at a given angle of incidence," Appl. Opt. 24, 1171-1179 (1985).

13. T. F. Thonn and R. M. A. Azzam, "Three-reflection halfwave and quarterwave retarders using dielectric-coated metallic mirrors," Appl. Opt. 23, 2752-2759 (1984).

14. R. M. A. Azzam and A. M. Kan'an, "In-line variable-phase reflection retarder using a coated deformable three-mirror system," Pure Appl. Opt. 3, 1-5 (1994). 